\title{
APPROXIMATELY MULTIPLICATIVE FUNCTIONALS ON ALGEBRAS OF SMOOTH FUNCTIONS
}

\author{
RICHARD ANDREW JONATHON HOWEY
}

\begin{abstract}
Let $\phi$ be a bounded linear functional on $A$, where $A$ is a commutative Banach algebra, then the bilinear functional $\check{\phi}$ is defined as $\check{\phi}(a, b)=\phi(a b)-\phi(a) \phi(b)$ for each $a$ and $b$ in $A$. If the norm of $\check{\phi}$ is small then $\phi$ is approximately multiplicative, and it is of interest whether or not $\|\check{\phi}\|$ being small implies that $\phi$ is near to a multiplicative functional. If this property holds for a commutative Banach algebra then $A$ is an AMNM algebra (approximately multiplicative functionals are near multiplicative functionals). The main result of the paper shows that $\mathrm{C}^{N}[0,1]^{M}$ (the complex valued functions defined on $[0,1]^{M}$ with all $N$ th order partial derivatives continuous) is AMNM. It is also shown that a similar proof can be applied to certain Lipschitz algebras.
\end{abstract}

\section{Introduction}

This paper is concerned with approximately multiplicative functionals on commutative Banach algebras. Let $\phi$ be a bounded linear functional on $A$, where $A$ is a commutative Banach algebra, then the bilinear functional $\check{\phi}$ is defined as $\check{\phi}(a, b)=\phi(a b)-\phi(a) \phi(b)$ for each $a$ and $b$ in $A$. If the norm of $\check{\phi}$ is small we say that $\phi$ is approximately multiplicative, and we are interested whether or not $\|\check{\phi}\|$ being small implies that $\phi$ is near to a multiplicative functional. If this property holds for a commutative Banach algebra we say that $A$ is an AMNM algebra (approximately multiplicative functionals are near multiplicative functionals).

Many of the classical commutative Banach algebras are shown to be AMNM in Johnson [4]. In this paper we show that the AMNM property holds for certain algebras of smooth functions. We will use an equivalent condition for the AMNM property for certain Banach algebras when the Gelfand and the norm topologies coincide on the character space of the algebra. The main result of the paper will be to show that $C^{N}[0,1]^{M}$ (the complex valued functions defined on $[0,1]^{M}$ with all $N$ th order partial derivatives continuous) is AMNM. Also we show that a similar proof can be applied to certain Lipschitz algebras. The work in this paper is taken from the author's thesis [2].

\section{Approximately multiplicative functionals}

Throughout this paper $A$ will denote a commutative Banach algebra, and $A^{\star}$ will denote the dual space of $A$. The set of characters of $A$, that is the nonzero multiplicative linear functionals on $A$, will be denoted by $\hat{A}$. The set of all multiplicative linear functionals on $A$ is thus $\hat{A} \cup\{0\}$. This section will be concerned with linear functionals on commutative Banach algebras. For each linear functional in the dual space of $A$ we define a bilinear functional on $A \times A$. 
Definition 2.1. For $\phi \in A^{\star}$ define $\check{\phi}$ by

$$
\check{\phi}(a, b)=\phi(a b)-\phi(a) \phi(b) \quad(a, b \in A) .
$$

If $\phi$ is a multiplicative linear functional then $\check{\phi}=0$. This gives us an idea of a linear functional $\phi$ being approximately multiplicative if we look at the norm of $\breve{\phi}$.

Definition 2.2. If $\|\check{\phi}\| \leqslant \delta$, where $\delta>0$, we say that $\phi$ is $\delta$-multiplicative.

An important result of Jarosz shows that approximately multiplicative functionals are continuous.

Proposition 2.3 (Jarosz [3, Proposition 5.5]). If $\phi$ is $\delta$-multiplicative for some $\delta \in \mathbb{R}^{+}$then $\phi$ is bounded and $\|\phi\| \leqslant 1+\delta$.

It is natural to ask if a linear functional being approximately multiplicative implies that it is near to a multiplicative linear functional. This leads us to the following definition for Banach algebras.

Definition 2.4. Let $A$ be a commutative Banach algebra. For each $\phi \in A^{\star}$ we put

$$
d(\phi)=\inf \{\|\phi-\psi\|: \psi \in \hat{A} \cup\{0\}\} .
$$

We say that $A$ is an algebra in which approximately multiplicative functionals are near multiplicative functionals, or $A$ is AMNM for short, if for each $\varepsilon>0$ there is $\delta>0$ such that if $\phi$ is a $\delta$-multiplicative linear functional then $d(\phi)<\varepsilon$.

It is often more convenient to think of the AMNM property in terms of sequences. The following proposition gives us some alternative definitions of AMNM.

Proposition 2.5 (Johnson [4, Proposition 3.2]). Let A be a unital Banach algebra. Then the following are equivalent.

(i) $A$ is $A M N M$.

(ii) For any sequence $\left\{\phi_{n}\right\}$ in $A^{\star}$ with $\left\|\check{\phi}_{n}\right\| \rightarrow 0$ there is a sequence $\left\{\psi_{n}\right\}$ in $\hat{A} \cup\{0\}$ with $\left\|\phi_{n}-\psi_{n}\right\| \rightarrow 0$.

(iii) For any sequence $\left\{\phi_{n}\right\}$ in $A^{\star}$ with $\left\|\check{\phi}_{n}\right\| \rightarrow 0$ there is a subsequence $\left\{\phi_{n_{i}}\right\}$ and a sequence $\left\{\psi_{i}\right\}$ in $\hat{A} \cup\{0\}$ with $\left\|\phi_{n_{i}}-\psi_{i}\right\| \rightarrow 0$.

Condition (iii) will be the preferred method to show that Banach algebras have the AMNM property.

Later we will consider $C^{N}[0,1]^{M}$, which is the algebra of complex valued functions defined on $[0,1]^{M}$ with all $N$ th order partial derivatives continuous. The norm of each $f \in \mathrm{C}^{N}[0,1]^{M}$ is given by

$$
\begin{aligned}
\|f\|= & \sup |f(x)| \\
& +\sum_{r=1}^{N} \frac{1}{r !}\left(\sum_{k_{1}+k_{2}+\ldots+k_{M}=r} \frac{r !}{k_{1} ! k_{2} ! \ldots k_{M} !} \sup \left|\frac{\partial^{r} f}{\partial x_{1}^{k_{1}} \partial x_{2}^{k_{2}} \ldots \partial x_{M}^{k_{M}}}(x)\right|\right) .
\end{aligned}
$$

For $\Omega$ a compact space we will also consider the $\operatorname{Lipschitz}$ algebras $\operatorname{Lip}(\Omega, d)$ and $\operatorname{lip}\left(\Omega, d^{\alpha}\right)(0<\alpha<1)$ which are defined as follows. 
Definition 2.6. Let $(\Omega, d)$ be a metric space and $f$ a complex valued function on $\Omega$. Then $f$ is a Lipschitz function if there exists a constant $\kappa$ such that

$$
|f(x)-f(y)| \leqslant \kappa d(x, y) \quad(x, y \in \Omega) .
$$

Definition 2.7. If $f$ is a Lipschitz function then the smallest $\kappa$ as in Definition 2.6 is the Lipschitz semi-norm, written $\|f\|_{d}$.

It is straightforward to show that

$$
\|f\|_{d}=\sup \left\{\frac{|f(x)-f(y)|}{d(x, y)}: x, y \in \Omega, x \neq y\right\} .
$$

The collection of bounded Lipschitz functions will be denoted by $\operatorname{Lip}(\Omega, d)$, which is a Banach algebra with norm defined by

$$
\|f\|=\|f\|_{\infty}+\|f\|_{d} \quad(f \in \operatorname{Lip}(\Omega, d)),
$$

where $\|f\|_{\infty}=\sup \{|f(x)|: x \in \Omega\}$.

Definition 2.8. Let $\operatorname{lip}(\Omega, d)$ be the subset of $\operatorname{Lip}(\Omega, d)$ of all functions $f$ in $\operatorname{Lip}(\Omega, d)$ such that

$$
\frac{|f(x)-f(y)|}{d(x, y)} \rightarrow 0 \quad \text { as } d(x, y) \rightarrow 0 .
$$

It can be shown that $\operatorname{lip}(\Omega, d)$ is a closed subalgebra of $\operatorname{Lip}(\Omega, d)$, and contains the constant functions. However, it may be the case that it only contains constant functions. (For example, take $\Omega=[0,1]$ and $d(x, y)=|x-y|$, then $f \in \operatorname{lip}(\Omega, d)$ implies that $f^{(1)}(x)=0$ for all $x$, so that $f$ is a constant.) To be assured of an abundance of non-constant functions, we shall consider the algebras $\operatorname{lip}\left(\Omega, d^{\alpha}\right), 0<\alpha<1$, where $d^{\alpha}$ is the metric on $\Omega$ defined by $d^{\alpha}(x, y)=[d(x, y)]^{\alpha}$ for $x, y \in \Omega$. The abundance of non-constant functions is shown later by Lemma 5.1.

In order to prove whether or not the AMNM property holds for a Banach algebra, it is useful to know how the multiplicative linear functionals are defined. The following proposition is a well-known result that will be used throughout the paper.

Proposition 2.9. Let $\Omega$ be a compact metric space and let $A$ be $\operatorname{Lip}(\Omega, d)$ or $\operatorname{lip}\left(\Omega, d^{\alpha}\right)(0<\alpha<1)$, or $C^{N}(\Omega)$ for $\Omega=[0,1]^{M}$. Then the non-zero multiplicative linear functionals in $A$ are given by maps $\psi$ such that

$$
\psi(f)=f(p) \quad(f \in A),
$$

for some unique $p \in \Omega$.

\section{When Gelfand and norm topologies coincide}

In this section we will see an equivalent condition for the ANMN property where the sequence tends towards a fixed multiplicative linear functional. Unfortunately, this is only for certain Banach algebras, namely separable unital Banach algebras where the Gelfand and norm topologies are the same on the character space of the algebra. 
THEOREM 3.1. Let $A$ be a separable unital Banach algebra where the Gelfand and norm topologies on $\hat{A}$ are the same. Then $A$ is AMNM if and only if

$$
\begin{aligned}
& \text { for all sequences }\left\{\phi_{n}\right\} \text { in } A^{\star} \text { with }\left\|\check{\phi}_{n}\right\| \rightarrow 0 \text { and } \phi_{n} \rightarrow \chi \text { weak* } \\
& \text { where } \chi \text { is a non-zero element of } A^{\star} \text { then }\left\|\phi_{n}-\chi\right\| \rightarrow 0 .
\end{aligned}
$$

Proof. To prove this theorem we will first show that (1) implies that $A$ is AMNM, then we will show the converse, that $A$ is AMNM implies (1).

First assume (1), then take $\left\{\phi_{n}\right\}$ in $A^{\star}$ with $\left\|\check{\phi}_{n}\right\| \rightarrow 0$. By Proposition $2.3,\left\{\phi_{n}\right\}$ is bounded, so let $\left\{\phi_{n}\right\}$ be bounded by $K$. Now we have $\left\{f: f \in A^{\star},\|f\| \leqslant K\right\}$ is weak* compact. Also the weak* topology on this set is metrizable, since $A$ is separable. Since $\left\{\phi_{n}\right\}$ is a sequence in a compact metric space it has a convergent subsequence. Thus we have a convergent subsequence in the weak* topology, so $\phi_{n_{i}} \rightarrow \chi$ weak*, say. Note that $\chi \in \hat{A} \cup\{0\}$ since $\left\|\breve{\phi}_{n_{i}}\right\| \rightarrow 0$. Either (i) $\chi \neq 0$ or (ii) $\chi=0$, and we consider these cases separately. For case (i) $\chi \neq 0$, we have by (1), $\left\|\phi_{n_{i}}-\chi\right\| \rightarrow 0$. For case (ii) $\chi(a)=0$ for all $a \in A$. Hence $\left|\phi_{n_{i}}(a)\right| \rightarrow 0$ for all $a \in A$.

Now

$$
\left\|\check{\phi}_{n_{i}}\right\|=\sup _{\|a\| \leqslant 1,\|b\| \leqslant 1}\left|\phi_{n_{i}}(a b)-\phi_{n_{i}}(a) \phi_{n_{i}}(b)\right|
$$

and putting $a=e$,

$$
\begin{aligned}
& \geqslant \sup _{\|b\| \leqslant 1}\left|\phi_{n_{i}}(b)-\phi_{n_{i}}(e) \phi_{n_{i}}(b)\right| \\
& =\left|1-\phi_{n_{i}}(e)\right|\left\|\phi_{n_{i}}\right\| .
\end{aligned}
$$

Thus $\left\|\phi_{n_{i}}\right\| \rightarrow 0$ since $\left\|\check{\phi}_{n_{i}}\right\| \rightarrow 0$ and $\left|1-\phi_{n_{i}}(e)\right| \rightarrow 1$. Hence $\left\|\phi_{n_{i}}-\chi\right\|=\left\|\phi_{n_{i}}\right\| \rightarrow 0$. Then by Proposition 2.5(iii), $A$ is AMNM.

Conversely suppose that $A$ is AMNM. Take a sequence $\left\{\phi_{n}\right\}$ in $A^{\star}$ with $\left\|\check{\phi}_{n}\right\| \rightarrow 0$ and $\phi_{n} \rightarrow \chi$ weak $^{*}(\chi \neq 0)$. We know that $A$ is AMNM, so there is a sequence $\left\{\psi_{n}\right\}$ in $\hat{A} \cup\{0\}$ with $\left\|\phi_{n}-\psi_{n}\right\| \rightarrow 0$. Thus $\phi_{n}-\psi_{n} \rightarrow 0$ weak*, therefore $\psi_{n} \rightarrow \chi$ weak*, which gives $\psi_{n} \rightarrow \chi$ in the Gelfand topology as $\psi_{n}, \chi \in \hat{A} \cup\{0\}$, and so $\left\|\psi_{n}-\chi\right\| \rightarrow 0$ as the Gelfand and norm topologies are equal.

Now

$$
\left\|\phi_{n}-\chi\right\| \leqslant\left\|\phi_{n}-\psi_{n}\right\|+\left\|\psi_{n}-\chi\right\| \rightarrow 0+0=0
$$

thus

$$
\left\|\phi_{n}-\chi\right\| \rightarrow 0
$$

4. $C^{N}[0,1]^{M}$ is $A M N M$

In this section we will prove that $\mathrm{C}^{N}[0,1]^{M}(N \in \mathbb{N}$ and $M \in \mathbb{N})$ has the AMNM property. We will also be looking at Lipschitz algebras and observing that a similar proof can be applied. We will be using Theorem 3.1 to show that $C^{N}[0,1]^{M}$ has the AMNM property. This algebra is separable since the polynomials are dense in $\mathrm{C}^{N}[0,1]^{M}$. Next we show that the Gelfand and the norm topologies are the same in $\mathrm{C}^{N}[0,1]^{M}$. 
Proposition 4.1. The Gelfand and norm topologies are equal in $\widehat{\mathrm{C}^{N}}[0,1]^{M}$.

Proof. Suppose that $F_{n} \rightarrow F$ in norm where $F_{n}, F \in \widehat{\mathrm{C}^{N}}[0,1]^{M}$. Then obviously $F_{n} \rightarrow F$ in the Gelfand topology.

Conversely, suppose that $F_{n} \rightarrow F$ in the Gelfand topology. By Proposition 2.9, for $G \in \widehat{\mathrm{C}^{N}}[0,1]^{M}, G(f)=f(x)$ for all $f \in \mathrm{C}^{N}[0,1]^{M}$ for some unique $x \in[0,1]^{M}$, so that

$$
f\left(y_{n}\right) \rightarrow f(y) \quad \text { for all } f \in C^{N}[0,1]^{M},
$$

where $y_{n}$ corresponds to $F_{n}$, and $y$ corresponds to $F$. For $i \in\{1,2, \ldots, M\}$ consider $f \in \mathrm{C}^{N}[0,1]^{M}$ defined by

$$
f(t)=t_{i} \quad\left(t=\left(t_{1}, t_{2}, \ldots, t_{M}\right) \in[0,1]^{M}\right) .
$$

Thus $\left(y_{n}\right)_{i} \rightarrow y_{i}$ for each $i \in\{1,2, \ldots, M\}$, that is $y_{n}$ converges pointwise. Now take $f \in \mathrm{C}^{N}[0,1]^{M}$ with $\|f\| \leqslant 1$. Then

$$
\left|f\left(y_{n}\right)-f(y)\right|=\left|\sum_{i=1}^{M} \frac{\partial f\left(z_{n}\right)}{\partial x_{i}}\left(y_{n}-y\right)_{i}\right|
$$

for some $z_{n}$ on the line segment from $y_{n}$ to $y$ by the mean value theorem, see Douglass [1, Chapter 8, Section 5]. Hence

$$
\begin{aligned}
\left|f\left(y_{n}\right)-f(y)\right| & \leqslant \sum_{i=1}^{M}\left|\frac{\partial f\left(z_{n}\right)}{\partial x_{i}}\right|\left|\left(y_{n}-y\right)_{i}\right| \\
& \leqslant \sum_{i=1}^{M}\left|\left(y_{n}-y\right)_{i}\right| .
\end{aligned}
$$

Thus

$$
\begin{aligned}
\left\|F_{n}-F\right\| & =\sup _{\|f\| \leqslant 1}\left|F_{n}(f)-F(f)\right| \\
& =\sup _{\|f\| \leqslant 1}\left|f\left(y_{n}\right)-f(y)\right| \\
& \leqslant \sup _{\|f\| \leqslant 1} \sum_{i=1}^{M}\left|\left(y_{n}-y\right)_{i}\right| \\
& =\sum_{i=1}^{M}\left|\left(y_{n}-y\right)_{i}\right| .
\end{aligned}
$$

Therefore $\left\|F_{n}-F\right\| \rightarrow 0$ since $\left(y_{n}\right)_{i} \rightarrow y_{i}$ for each $i \in\{1,2, \ldots, M\}$.

To show that $\mathrm{C}^{N}[0,1]^{M}$ is $\mathrm{AMNM}$, we need an extension result regarding elements in $C^{N}[0,1]^{M}$. First we define a restriction norm on $C^{N}[0,1]^{M}$.

Definition 4.2. For $C^{N}[0,1]^{M}$ and Lipschitz algebras, let the restriction norm over $B$, denoted $\|f\|^{B}$, be the usual norm of the restriction of $f$ to $B$; that is for 
$C^{N}[0,1]^{M}$ and the set $B \subseteq[0,1]^{M}$ we have

$$
\begin{aligned}
\|f\|^{B}= & \sup _{x \in B}|f(x)| \\
& +\sum_{r=1}^{N} \frac{1}{r !}\left(\sum_{k_{1}+k_{2}+\ldots+k_{M}=r} \frac{r !}{k_{1} ! k_{2} ! \ldots k_{M} !} \sup _{x \in B}\left|\frac{\partial^{r} f}{\partial x_{1}^{k_{1}} \partial x_{2}^{k_{2}} \ldots \partial x_{M}^{k_{M}}}(x)\right|\right) .
\end{aligned}
$$

Proposition 4.3. There exists $L \in \mathbb{R}^{+}$(which depends on $N$ and $M$ only) such that for a closed Euclidean ball $B \subseteq[0,1]^{M}$ and $f \in \mathrm{C}^{N}[0,1]^{M}$ with

$$
\|f\|^{B}<\eta \quad(0<\eta<1)
$$

there exists $h \in \mathrm{C}^{N}[0,1]^{M}$ with $h(x)=f(x)$ for all $x \in B$ and $\|h\| \leqslant L \eta$.

Proof. By Whitney [7, Theorem 1] a suitable extension can be found. It is necessary to refer to Whitney [6, in particular Section 10 and Section 11] to see that our constant $L$ satisfies the required conditions.

To show that $C^{N}[0,1]^{M}$ is AMNM we will also use the fact that $C^{N}[0,1]^{M}$ is a regular Banach algebra; the definition of a regular Banach algebra is shown below.

Definition 4.4. A Banach algebra $A$ is a regular Banach algebra if it is semisimple, and for any $p \in \hat{A}$ and closed $B \subset \hat{A}$ with $p \notin B$ there exists $a \in A$ such that $\hat{A}(p) \neq 0$ and $\hat{A}(f)=0$ for all $f \in B$.

THEOREM 4.5. $\mathrm{C}^{N}[0,1]^{M}$ is $A M N M$.

Proof. We will use Theorem 3.1 to prove that $C^{N}[0,1]^{M}$ is AMNM. First we will let $\left\{\phi_{n}\right\}$ be a sequence in $\left(\mathrm{C}^{N}[0,1]^{M}\right)^{\star}$ with $\left\|\check{\phi}_{n}\right\| \rightarrow 0$ and $\phi_{n} \rightarrow \chi$ weak $^{*}$ where $\chi(f)=f(p)$ for some $p \in[0,1]^{M}$. Then we will prove successively the following claims.

(i) For any closed Euclidean ball $B \subseteq[0,1]^{M}$ with $p \in B \backslash \partial B$ we have

$$
\sup _{\|g\| \leqslant 1}\left\{\left|\phi_{n}(g)\right|: g(x)=0 \text { for all } x \in B\right\} \rightarrow 0 \quad \text { as } n \rightarrow \infty .
$$

(ii) For any closed ball $B$ with $p \in B \backslash \partial B, 0<\eta<1$, and $\varepsilon>0$,

there exists $N_{0}$ such that

$$
\sup _{\|f\| \leqslant 1,\|f\|^{B} \leqslant \eta}\left|\phi_{n}(f)\right|<\frac{\varepsilon}{2}+K_{0} \eta \quad \text { for } n \geqslant N_{0},
$$

where $K_{0}$ is some constant depending on $N$ and $M$ only.

(iii) $\sup _{\|f\| \leqslant 1}\left\{\left|\phi_{n}\left(f^{N+1}\right)\right|: f(p)=0\right\} \rightarrow 0$ as $n \rightarrow \infty$.

(iv) $\sup _{\|f\| \leqslant 1}\left\{\left|\phi_{n}(f)\right|: f(p)=0\right\} \rightarrow 0$ as $n \rightarrow \infty$.

(v) $\left\|\phi_{n}-\chi\right\| \rightarrow 0$ as $n \rightarrow \infty$.

Set $A=C^{N}[0,1]^{M}$ and let $\left\{\phi_{n}\right\}$ be a sequence in $A^{\star}$ with $\left\|\check{\phi}_{n}\right\| \rightarrow 0$ and $\phi_{n} \rightarrow$ $\chi$ weak $^{*}$, where $\chi(f)=f(p)$ for all $f \in A$ for some $p \in[0,1]^{M}$. Thus $\left|\phi_{n}(f)-f(p)\right|$ $\rightarrow 0$ for all $f \in A$, and putting $f=1$ gives

$$
\left|\phi_{n}(1)-1\right| \rightarrow 0 \text {. }
$$


Now take a closed Euclidean ball $B \subseteq[0,1]^{M}$ with $p \in B \backslash \partial B$. Then

$$
\begin{aligned}
\left\|\check{\phi}_{n}\right\|= & \sup _{\|f\| \leqslant 1,\|g\| \leqslant 1}\left|\phi_{n}(f g)-\phi_{n}(f) \phi_{n}(g)\right| \\
\geqslant & \sup _{\|f\| \leqslant 1,\|g\| \leqslant 1}\left\{\left|\phi_{n}(f) \| \phi_{n}(g)\right|: g(x)=0 \text { for all } x \in B,\right. \\
& \left.f(x)=0 \text { for all } x \in[0,1]^{M} \backslash B\right\} .
\end{aligned}
$$

Since $A$ is regular, we can choose $f_{0} \in A$ with $f_{0}(x)=0$ for $x \in[0,1]^{M} \backslash B$ such that $f_{0}(p) \neq 0$ and $\left\|f_{0}\right\| \leqslant 1$, and so

$$
\left\|\check{\phi}_{n}\right\| \geqslant\left|\phi_{n}\left(f_{0}\right)\right| \sup _{\|g\| \leqslant 1}\left\{\left|\phi_{n}(g)\right|: g(x)=0 \text { for all } x \in B\right\} .
$$

Thus

$$
\sup _{\|g\| \leqslant 1}\left\{\left|\phi_{n}(g)\right|: g(x)=0 \text { for all } x \in B\right\} \rightarrow 0
$$

since $\left\|\check{\phi}_{n}\right\| \rightarrow 0$ and $\phi_{n}\left(f_{0}\right) \rightarrow f_{0}(p) \neq 0$.

By Proposition 2.3, $\left\{\phi_{n}\right\}$ is a bounded sequence, so we can let $K=\sup _{n}\left\|\phi_{n}\right\|$.

Now take $f$ such that $\|f\| \leqslant 1$ with $\|f\|^{B}<\eta \quad(0<\eta<1)$. By Proposition 4.3, it is possible to define an extension $h$ of $f$ such that $h(x)=f(x)$ for all $x$ in $B$ and $\|h\| \leqslant L \eta$ for some constant $L$ depending on $N$ and $M$ only.

Let $g=f-h$, so $g \in A$ and $g(x)=0$ for $x \in B$ and

$$
\|g\|=\|f-h\| \leqslant\|f\|+\|h\| \leqslant 1+L \eta<1+L .
$$

This gives

$$
\begin{aligned}
\sup _{\|f\| \leqslant 1,\|f\|^{B} \leqslant \eta}\left|\phi_{n}(f)\right|= & \sup _{\{}\left\{\left|\phi_{n}(g+h)\right|: g \text { and } h \text { are given by } f \text { as above }\right\} \\
\leqslant & \sup _{\|g\| \leqslant 1+L}\left\{\left|\phi_{n}(g)\right|: g(x)=0 \text { for all } x \in B\right\} \\
& +\sup _{\|h\|<L \eta}\left|\phi_{n}(h)\right| .
\end{aligned}
$$

Let $\varepsilon>0$, then by (3) there exists $N_{0}$ so that $\sup _{\|g\| \leqslant 1+L}\left\{\left|\phi_{n}(g)\right|: g(x)=0\right.$ for $\left.\in B\right\}$ $<\varepsilon / 2$ for $n \geqslant N_{0}$, so that the above is less than

$$
\begin{aligned}
\frac{\varepsilon}{2}+ & \sup _{\|h\|<L \eta}\left\|\phi_{n}\right\|\|h\| \quad \text { for } n \geqslant N_{0} \\
& <\frac{\varepsilon}{2}+K L \eta .
\end{aligned}
$$

Thus we have, for any closed ball $B$ with $p \in B \backslash \partial B, 0<\eta<1$, and $\varepsilon>0$,

there exists $N_{0}$ such that

$$
\sup _{\|f\| \leqslant 1,\|f\|^{B} \leqslant \eta}\left|\phi_{n}(f)\right|<\frac{\varepsilon}{2}+L K \eta \quad \text { for } n \geqslant N_{0} .
$$

Now take $\varepsilon$ such that

$$
0<\varepsilon<2 L K<2 L(N !)(N+1)^{((N+2) M+1)} K,
$$

and let $\delta$ be such that

$$
0<\delta<\frac{\varepsilon}{2 L(N !)(N+1)^{((N+2) M+1)} K} .
$$


Let $B$ be the closed ball centre $p$, radius $\delta / M$. Take $x \in B$ and $f \in A$ such that $f(p)=0$ and $\|f\| \leqslant 1$. Then

$$
|f(x)-f(p)|=\left|\sum_{i=1}^{M} \frac{\partial f\left(x^{\prime}\right)}{\partial x_{i}}(x-p)_{i}\right|
$$

for some $x^{\prime}$ on the line segment from $x$ to $p$ by the mean value theorem, see Douglass [1, Chapter 8, Section 5]. Thus

$$
\begin{aligned}
|f(x)|=|f(x)-f(p)| & \leqslant \sum_{i=1}^{M}\left|\frac{\partial f\left(x^{\prime}\right)}{\partial x_{i}}\right|\left|(x-p)_{i}\right| \\
& \leqslant \sum_{i=1}^{M}\left|(x-p)_{i}\right| \\
& \leqslant M\left(\sum_{i=1}^{M}\left|(x-p)_{i}\right|^{2}\right)^{1 / 2} \\
& \leqslant M \frac{\delta}{M}=\delta,
\end{aligned}
$$

and taking the supremum over $B$ we have

$$
\sup _{x \in B}|f(x)| \leqslant \delta .
$$

Now take $x \in B, r \in\{0,1,2, \ldots, N\}$ and $k_{1}, k_{2}, \ldots, k_{M} \in \mathbb{N} \cup\{0\}$ such that $\sum_{i=1}^{M} k_{i}=r$, then a direct calculation shows that

$$
\left|\frac{\partial^{r}}{\partial x_{1}{ }^{k_{1}} \partial x_{2}{ }^{k_{2}} \ldots \partial x_{M}{ }^{k_{M}}}(f(x))^{N+1}\right| \leqslant \frac{\varepsilon}{2 L(N+1)^{(M+1)} K},
$$

and taking the supremum over $B$ we have

$$
\sup _{x \in B}\left|\frac{\partial^{r}}{\partial x_{1}{ }^{k_{1}} \partial x_{2}{ }^{k_{2}} \ldots \partial x_{M}{ }^{k_{M}}}(f(x))^{N+1}\right| \leqslant \frac{\varepsilon}{2 L(N+1)^{(M+1)} K} .
$$

This then gives, after another calculation,

$$
\left\|f^{N+1}\right\|^{B}<\frac{\varepsilon}{2 L K}
$$

Also

$$
\left\|f^{N+1}\right\| \leqslant\|f\|^{N+1} \leqslant 1
$$

Thus we have by (4) for some $N_{0}$

$$
\begin{aligned}
\sup _{\|f\| \leqslant 1}\left\{\left|\phi_{n}\left(f^{N+1}\right)\right|: f(p)=0\right\} & \leqslant \sup _{\|f\| \leqslant 1}\left\{\left|\phi_{n}(f)\right|:\|f\|^{B} \leqslant \frac{\varepsilon}{2 L K}\right\} \\
& <\frac{\varepsilon}{2}+L K \frac{\varepsilon}{2 L K}=\varepsilon \quad \text { for } n \geqslant N_{0} .
\end{aligned}
$$

Therefore

$$
\sup _{\|f\| \leqslant 1}\left\{\left|\phi_{n}\left(f^{N+1}\right)\right|: f(p)=0\right\} \rightarrow 0 \quad \text { as } n \rightarrow \infty .
$$


Now

$$
\begin{aligned}
\mid \check{\phi}_{n}( & \left.f^{N}, f\right)+\check{\phi}_{n}\left(f^{N-1}, f\right) \phi_{n}(f)+\check{\phi}_{n}\left(f^{N-2}, f\right)\left(\phi_{n}(f)\right)^{2} \\
& +\ldots+\check{\phi}_{n}(f, f)\left(\phi_{n}(f)\right)^{N-1} \mid \\
= & \mid \phi_{n}\left(f^{N+1}\right)-\phi_{n}\left(f^{N}\right) \phi_{n}(f)+\phi_{n}\left(f^{N}\right) \phi_{n}(f)-\phi_{n}\left(f^{N-1}\right)\left(\phi_{n}(f)\right)^{2} \\
& +\ldots+\phi_{n}\left(f^{2}\right)\left(\phi_{n}(f)\right)^{N-1}-\left(\phi_{n}(f)\right)^{N+1} \mid \\
= & \left|\phi_{n}\left(f^{N+1}\right)-\left(\phi_{n}(f)\right)^{N+1}\right|,
\end{aligned}
$$

so taking the supremum over $\|f\| \leqslant 1$ with $f(p)=0$ we get

$$
\begin{aligned}
\sup _{\|f\| \leqslant 1}\{ & \left.\left|\phi_{n}\left(f^{N+1}\right)-\left(\phi_{n}(f)\right)^{N+1}\right|: f(p)=0\right\} \\
= & \sup _{\|f\| \leqslant 1}\left\{\check{\phi}_{n}\left(f^{N}, f\right)+\check{\phi}_{n}\left(f^{N-1}, f\right) \phi_{n}(f)+\check{\phi}_{n}\left(f^{N-2}, f\right)\left(\phi_{n}(f)\right)^{2}\right. \\
& \left.\quad+\ldots+\check{\phi}_{n}(f, f)\left(\phi_{n}(f)\right)^{N-1} \mid: f(p)=0\right\} .
\end{aligned}
$$

Therefore since $\left\|\check{\phi}_{n}\right\| \rightarrow 0$ and $\left\{\phi_{n}\right\}$ is bounded

$$
\sup _{\|f\| \leqslant 1}\left\{\left|\phi_{n}\left(f^{N+1}\right)-\left(\phi_{n}(f)\right)^{N+1}\right|: f(p)=0\right\} \rightarrow 0 \quad \text { as } n \rightarrow \infty .
$$

Then by (5) we have

$$
\sup _{\|f\| \leqslant 1}\left\{\left|\left(\phi_{n}(f)\right)^{N+1}\right|: f(p)=0\right\} \rightarrow 0 \quad \text { as } n \rightarrow \infty .
$$

Thus

$$
\sup _{\|f\| \leqslant 1}\left\{\left|\phi_{n}(f)\right|: f(p)=0\right\} \rightarrow 0 \quad \text { as } n \rightarrow \infty .
$$

Now $A=\{f \in A: f(p)=0\}+\mathbb{C}$ so that for each $g \in A$ we can write $g=g_{0}+c$ for some $g_{0} \in\{f \in A: f(p)=0\}$ and $c \in \mathbb{C}$, thus

$$
\begin{aligned}
\left\|\phi_{n}-\chi\right\| & =\sup _{\|g\| \leqslant 1}\left|\phi_{n}(g)-g(p)\right| \\
& =\sup _{\|g\| \leqslant 1}\left|\phi_{n}\left(g_{0}+c\right)-g_{0}(p)-c\right| \\
& \leqslant \sup _{\|g\| \leqslant 1}\left\{\left|\phi_{n}(g)\right|: g(p)=0\right\}+\left|\phi_{n}(1)-1\right| .
\end{aligned}
$$

Therefore by (2) and (6)

$$
\left\|\phi_{n}-\chi\right\| \rightarrow 0 \quad \text { as } n \rightarrow \infty .
$$

Thus we have for each sequence $\left\{\phi_{n}\right\}$ in $A^{\star}$ with $\left\|\check{\phi}_{n}\right\| \rightarrow 0$ and $\phi_{n} \rightarrow \chi$ weak $^{*}$ where $\chi$ is a non-zero element of $A^{\star}$, that $\left\|\phi_{n}-\chi\right\| \rightarrow 0$. Hence by Theorem 3.1, $A$ is AMNM.

\section{5. $\operatorname{Lip}(X, d)$ and $\operatorname{lip}\left(X, d^{\alpha}\right)$ are $A M N M$}

Throughout this section $(X, d)$ will be a compact metric space. We will show how similar techniques can be applied to Lipschitz algebras to prove the AMNM property, and that the techniques are easier to apply in this instance. Again, we 
will be using Theorem 3.1 to show that the Lipschitz algebras have the AMNM property. Again, we will show that the Gelfand and the norm topologies are the same. First we have a useful result for Lipschitz algebras.

Lemma 5.1. Consider the Banach algebras $\operatorname{Lip}(X, d)$ and $\operatorname{lip}\left(X, d^{\alpha}\right)(0<\alpha<1$, $\left.d^{\alpha}(x, y)=[d(x, y)]^{\alpha}\right)$, where $X$ has finite diameter $d^{\prime}$; then the function $f$ defined by

$$
f(x)=\min \{d(x, t), 1\} \quad(x \in X)
$$

for some $t \in X$, is an element. Also $\|f\|_{\infty} \leqslant d^{\prime},\|f\|_{d} \leqslant 1$ and $\|f\|_{d^{\alpha}} \leqslant\left(d^{\prime}\right)^{1-\alpha}$.

Proof. This is straightforward.

Proposition 5.2. The Gelfand and the norm topologies are equal in $\widehat{\operatorname{Lip}}(X, d)$ and $\widehat{\operatorname{lip}}\left(X, d^{\alpha}\right)$ for $0<\alpha<1$ where $\widehat{\operatorname{Lip}}(X, d)$ and $\widehat{\operatorname{lip}}\left(X, d^{\alpha}\right)$ are separable.

Proof. Let $A$ denote $\widehat{\operatorname{Lip}}(X, d)$ or $\widehat{\operatorname{lip}}\left(X, d^{\alpha}\right)$ for some $0<\alpha<1$.

Suppose that $F_{n} \rightarrow F$ in norm where $F_{n}, F \in \hat{A}$. Then obviously we have $F_{n} \rightarrow F$ in the Gelfand topology.

Conversely, suppose that $F_{n} \rightarrow F$ in the Gelfand topology so that by Proposition 2.9

$$
f\left(y_{n}\right) \rightarrow f(y) \quad \text { for all } f \in A
$$

where $y_{n}$ corresponds to $F_{n}$, and $y$ corresponds to $F$. Consider $f \in A$ defined by $f(x)=\min \{d(x, y), 1\}$ from Lemma 5.1, which gives $f\left(y_{n}\right) \rightarrow d(y, y)$, so that $d\left(y_{n}, y\right) \rightarrow 0$.

Now if $A$ is $\widehat{\operatorname{Lip}}(X, d)$ then

$$
\begin{aligned}
& \left\|F_{n}-F\right\|=\sup _{\|f\| \leqslant 1}\left|F_{n}(f)-F(f)\right| \\
& =\sup _{\|f\| \leqslant 1}\left|f\left(y_{n}\right)-f(y)\right| \\
& \leqslant \sup _{\|f\| \leqslant 1}\|f\|_{d} d\left(y_{n}, y\right) \\
& \text { (Since }\|f\|_{d}=\sup _{x^{\prime} \neq y^{\prime}} \frac{\left|f\left(x^{\prime}\right)-f\left(y^{\prime}\right)\right|}{d\left(x^{\prime}, y^{\prime}\right)} \text {, so }\|f\|_{d} \geqslant \frac{\left|f\left(y_{n}\right)-f(y)\right|}{d\left(y_{n}, y\right)} \text { if } y_{n} \neq y \text {.) } \\
& \leqslant d\left(y_{n}, y\right) \text {. }
\end{aligned}
$$

Thus $\left\|F_{n}-F\right\| \rightarrow 0$ since $d\left(y_{n}, y\right) \rightarrow 0$. A similiar argument applies in the case when $A$ is $\widehat{\operatorname{lip}}\left(X, d^{\alpha}\right)$.

Again, we need an extension result to show that the Lipschitz algebras have the AMNM property. The following result is due to $\operatorname{McSh}$ ane for extending in $\operatorname{Lip}(X, d)$, but unfortunately does not seem to apply to $\operatorname{lip}\left(X, d^{\alpha}\right)$, although we will show an extension result for $\operatorname{lip}\left(X, d^{\alpha}\right)$ when $X=[0,1]$ and $d^{\alpha}(x, y)=|x-y|^{\alpha}$ for $0<\alpha<1$.

THEOREM 5.3 (McShane [5, Theorem 1]). Let the real valued function $f$ be defined on a subset $E$ of the metric space $(X, d)$ satisfying the Lipschitz condition

$$
|f(x)-f(y)| \leqslant K d(x, y) \quad(x, y \in E)
$$

on $E$ for some $K \in \mathbb{R}^{+}$. Then $f$ can be extended to $X$ preserving the same Lipschitz condition. 
Summary of proof. Define $h$ to be the real valued function on $X$ given by

$$
h(x)=\sup _{y \in E}\{f(y)-K d(x, y)\} .
$$

Then it can easily be shown that $h$ satisfies the conditions for the extension of $f$.

COROLlary 5.4. Let $f$ be the complex valued Lipschitz function defined on a subset $E$ of metric space $(X, d)$ with $\|f\|^{E}<\eta$. Then there exists $h \in \operatorname{Lip}(X, d)$ such that $\|h\|<2 \eta$ and $h(x)=f(x)$ for each $x \in E$.

Proof. This is straightforward.

Lemma 5.5. For an interval $I=\left[l_{1}, l_{2}\right]$ in $[0,1]$ and $f \in \operatorname{lip}\left([0,1], d^{\alpha}\right)$, where $d^{\alpha}(x, y)=|x-y|^{\alpha}$ for $0<\alpha<1$ with $\|f\| \leqslant 1$ and

$$
\|f\|^{I}<\eta \quad(0<\eta<1),
$$

there exists $h \in \operatorname{lip}\left([0,1], d^{\alpha}\right)$ with $h(x)=f(x)$ for all $x \in I$ and $\|h\| \leqslant \eta$.

Proof. Take $f \in \operatorname{lip}\left([0,1], d^{\alpha}\right)$ with $\|f\| \leqslant 1$ and $\|f\|^{I}<\eta$ for some $0<\eta<1$ and interval $I=\left[l_{1}, l_{2}\right]$ in $[0,1]$. Let $h \in \operatorname{lip}\left([0,1], d^{\alpha}\right)$ be defined by

$$
h(x)= \begin{cases}f\left(l_{1}\right) & 0 \leqslant x \leqslant l_{1} \\ f(x) & l_{1} \leqslant x \leqslant l_{2} \\ f\left(l_{2}\right) & l_{2} \leqslant x \leqslant 1\end{cases}
$$

Then $h$ is easily shown to satisfy the required conditions.

Next we show that the Lipschitz algebras which satisfy certain conditions are AMNM using Theorem 3.1.

Proposition 5.6. Let $A$ be a separable Lipschitz algebra $\operatorname{Lip}(X, d)$ or $\operatorname{lip}\left(X, d^{\alpha}\right)$ where $(X, d)$ is not the discrete metric. Also suppose there exists $L \in \mathbb{R}^{+}$such that for any closed ball $B \subseteq X$ and $f \in A$ with $\|f\| \leqslant 1$ and $\|f\|^{B}<\eta(0<\eta<1)$ then there exists $h \in A$ with $h(x)=f(x)$ for each $x \in B$ and $\|h\| \leqslant L \eta$. Then $A$ is AMNM.

Proof. Let $A$ be the algebra in question. Then first we will let $\left\{\phi_{n}\right\}$ be a sequence

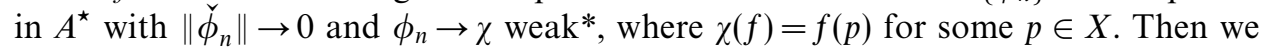
will prove successively the following claims.

(i) For any closed ball $B \neq X$ in $X$ with $p \in B \backslash \partial B$ we have

$$
\sup _{\|g\| \leqslant 1}\left\{\left|\phi_{n}(g)\right|: g(x)=0 \text { for all } x \in B\right\} \rightarrow 0 \quad \text { as } n \rightarrow \infty .
$$

(ii) For any closed ball $B$ with $p \in B \backslash \partial B, 0<\eta<1$, and $\varepsilon>0$ :

there exists $N$ such that

where $K=\sup _{n}\left\|\phi_{n}\right\|$.

$$
\sup _{\|f\| \leqslant 1,\|f\|^{B} \leqslant \eta}\left|\phi_{n}(f)\right|<\frac{\varepsilon}{2}+L K \eta \quad \text { for } n \geqslant N,
$$

(iii) $\sup _{\|f\| \leqslant 1}\left\{\left|\phi_{n}\left(f^{2}\right)\right|: f(p)=0\right\} \rightarrow 0$ as $n \rightarrow \infty$.

(iv) $\sup _{\|f\| \leqslant 1}\left\{\left|\phi_{n}(f)\right|: f(p)=0\right\} \rightarrow 0$ as $n \rightarrow \infty$.

(v) $\left\|\phi_{n}-\chi\right\| \rightarrow 0$ as $n \rightarrow \infty$. 
Let $\left\{\phi_{n}\right\}$ be a sequence in $A^{\star}$ with $\left\|\check{\phi}_{n}\right\| \rightarrow 0$ and $\phi_{n} \rightarrow \chi$ weak $^{*}$, where $\chi(f)=f(p)$ for some unique $p \in X$ by Proposition 2.9. By the weak* convergence we have

$$
\left|\phi_{n}(f)-f(p)\right| \rightarrow 0 \quad \text { for all } f \in A .
$$

Putting $f=1$ gives

$$
\left|\phi_{n}(1)-1\right| \rightarrow 0
$$

Now take a closed ball $B \neq X$ in $X$ with $p \in B \backslash \partial B$. Then

$$
\begin{aligned}
\left\|\check{\phi}_{n}\right\|= & \sup _{\|f\| \leqslant 1,\|g\| \leqslant 1}\left|\phi_{n}(f g)-\phi_{n}(f) \phi_{n}(g)\right| \\
\geqslant & \sup _{\|f\| \leqslant 1,\|g\| \leqslant 1}\left\{\left|\phi_{n}(f)\right|\left|\phi_{n}(g)\right|: g(x)=0 \text { for } \in B,\right. \\
& f(x)=0 \text { for } \in X \backslash B\} .
\end{aligned}
$$

Since $A$ is regular we can choose $f_{0} \in A$ such that $f_{0}(p) \neq 0, f_{0}(x)=0$ for $x \in X \backslash B$ and $\left\|f_{0}\right\| \leqslant 1$, and so

$$
\left\|\check{\phi}_{n}\right\| \geqslant\left|\phi_{n}\left(f_{0}\right)\right| \sup _{\|g\| \leqslant 1}\left\{\left|\phi_{n}(g)\right|: g(x)=0 \text { for } \in B\right\} .
$$

Thus

$$
\sup _{\|g\| \leqslant 1}\left\{\left|\phi_{n}(g)\right|: g(x)=0 \text { for } \in B\right\} \rightarrow 0
$$

since $\left\|\check{\phi}_{n}\right\| \rightarrow 0$ and $\phi_{n}\left(f_{0}\right) \rightarrow f_{0}(p) \neq 0$.

By Proposition 2.3, $\left\{\phi_{n}\right\}$ is a bounded sequence, so we can let $K=\sup _{n}\left\|\phi_{n}\right\|$.

Now take $f \in A$ with $\|f\| \leqslant 1$ and $\|f\|^{B}<\eta$ for some $0<\eta<1$ and closed ball $B$ in $X$. Then there exists $h \in A$ with $h(x)=f(x)$ for all $x \in B$ and $\|h\| \leqslant L \eta$. Let $g \in A$ be given by $g=f-h$, so that $g(x)=0$ for $x \in B$, and $\|g\|=\|f-h\| \leqslant\|f\|+\|h\| \leqslant$ $1+L \eta<1+L$. This gives

$$
\begin{aligned}
\sup _{\|f\| \leqslant 1,\|f\|^{B} \leqslant \eta}\left|\phi_{n}(f)\right| & =\sup \left\{\left|\phi_{n}(g+h)\right|: g \text { and } h \text { are given by } f \text { as above }\right\} \\
& \leqslant \sup _{\|g\| \leqslant 1+L}\left\{\left|\phi_{n}(g)\right|: g(x)=0 \text { for } \in B\right\}+\sup _{\|h\|<\eta}\left|\phi_{n}(h)\right| .
\end{aligned}
$$

By (8) there exists $N$ such that $\sup _{\|g\| \leqslant 1+L}\left\{\left|\phi_{n}(g)\right|: g(x)=0\right.$ for $\left.\in B\right\}<\varepsilon / 2$ for $n \geqslant N$, so that

$$
\begin{aligned}
\sup _{\|f\| \leqslant 1,\|f\|^{B} \leqslant \eta}\left|\phi_{n}(f)\right| & <\frac{\varepsilon}{2}+\sup _{\|h\|<L \eta}\left\|\phi_{n}\right\|\|h\| & \text { for } n \geqslant N \\
& <\frac{\varepsilon}{2}+L K \eta & \text { for } n \geqslant N .
\end{aligned}
$$

Thus we have for any closed ball $B$, with $p \in B \backslash \partial B, 0<\eta<1$, and $\varepsilon>0$,

$$
\text { there exists } N \text { such that } \sup _{\|f\| \leqslant 1,\|f\|^{B} \leqslant \eta}\left|\phi_{n}(f)\right|<\frac{\varepsilon}{2}+L K \eta \quad \text { for } n \geqslant N \text {. }
$$

Now take $0<\varepsilon<2 L K$ and $f \in A$ with $f(p)=0$ and $\|f\| \leqslant 1$.

Let $\delta$ be such that

$$
0<\delta<\frac{\varepsilon}{8 L K}
$$

and let $B$ be the closed ball given by

$$
\{x: x \in X, d(x, p) \leqslant \delta\} .
$$


We will now consider the square $f^{2}$ of $f$ on $B$ and show that $\left\|f^{2}\right\|^{B}<\varepsilon / 2 L K$ for the case $A=\operatorname{Lip}(X, d)$. If $A=\operatorname{lip}\left(X, d^{\alpha}\right)$ then it can be shown by a similar method that $\left\|f^{2}\right\|^{B}<\varepsilon / 2 L K$.

Since $f$ is continuous, there exists a point $c \in B$ such that $|f(c)|=\sup _{x \in B}|f(x)|$. We have $\|f\|^{B}=\|f\|_{\infty}^{B}+\|f\|_{d}^{B} \leqslant 1$, thus

$$
\sup _{x, y \in B, x \neq y}\left|\frac{f(x)-f(y)}{d(x, y)}\right| \leqslant 1 .
$$

If $|f(c)| \neq 0$ then $c \neq p$ and so

$$
\left|\frac{f(c)-f(p)}{d(c, p)}\right| \leqslant 1,
$$

thus

$$
\sup _{x \in B}|f(x)|=|f(c)| \leqslant d(c, p) \leqslant \delta<\frac{\varepsilon}{8 L K} .
$$

Therefore

$$
\sup _{x \in B}\left|f^{2}(x)\right|<\left(\frac{\varepsilon}{8 L K}\right)^{2}<\frac{\varepsilon}{8 L K}
$$

since $\|f\| \leqslant 1$ and $\varepsilon / 8 L K<1$. Next we look at the Lipschitz norm of $f^{2}$ restricted to $B$ :

$$
\begin{aligned}
\left\|f^{2}\right\|_{d}^{B}= & \sup _{x, y \in B, x \neq y}\left|\frac{f^{2}(x)-f^{2}(y)}{d(x, y)}\right| \\
= & \sup _{x, y \in B, x \neq y}\left|\frac{f(x)-f(y)}{d(x, y)}\right||f(x)+f(y)| \\
& \leqslant\|f\|_{d} \times 2 \sup _{x \in B}|f(x)|<1 \times 2 \times \frac{\varepsilon}{8 L K}=\frac{\varepsilon}{4 L K} .
\end{aligned}
$$

Therefore the norm of $f^{2}$ satisfies

$$
\left\|f^{2}\right\|^{B}=\left\|f^{2}\right\|_{\infty}^{B}+\left\|f^{2}\right\|_{d}^{B}<\frac{\varepsilon}{8 L K}+\frac{\varepsilon}{4 L K}=\frac{3 \varepsilon}{8 L K}<\frac{\varepsilon}{2 L K} .
$$

Therefore by (9) there exists $N$ such that

$$
\begin{aligned}
\sup _{\|f\| \leqslant 1}\left\{\left|\phi_{n}\left(f^{2}\right)\right|: f(p)=0\right\} & \leqslant \sup _{\|f\| \leqslant 1,\|f\|^{B}<\varepsilon / 2 L K}\left|\phi_{n}(f)\right| \\
& <\frac{\varepsilon}{2}+L K\left(\frac{\varepsilon}{2 L K}\right)=\varepsilon \quad \text { for } n \geqslant N
\end{aligned}
$$

thus

$$
\sup _{\|f\| \leqslant 1}\left\{\left|\phi_{n}\left(f^{2}\right)\right|: f(p)=0\right\} \rightarrow 0
$$

Now

$$
\left\|\check{\phi}_{n}\right\| \geqslant \sup _{\|f\| \leqslant 1}\left\{\left|\phi_{n}\left(f^{2}\right)-\phi_{n}(f)^{2}\right|: f(p)=0\right\},
$$

therefore

$$
\sup _{\|f\| \leqslant 1}\left\{\left|\phi_{n}\left(f^{2}\right)-\phi_{n}(f)^{2}\right|: f(p)=0\right\} \rightarrow 0
$$


since $\left\|\check{\phi}_{n}\right\| \rightarrow 0$, so by (10) we have

$$
\sup _{\|f\| \leqslant 1}\left\{\left|\phi_{n}(f)\right|: f(p)=0\right\} \rightarrow 0 .
$$

Now $A=\{f \in A: f(p)=0\}+\mathbb{C}$ so that for each $g \in A$ we can write $g=g_{0}+c$ for some $g_{0} \in\{f \in A: f(p)=0\}$ and $c \in \mathbb{C}$, thus

$$
\begin{aligned}
\left\|\phi_{n}-\chi\right\| & =\sup _{\|g\| \leqslant 1}\left|\phi_{n}(g)-g(p)\right| \\
& =\sup _{\|g\| \leqslant 1}\left|\phi_{n}\left(g_{0}+c\right)-g_{0}(p)-c\right| \\
& \leqslant \sup _{\|g\| \leqslant 1}\left\{\left|\phi_{n}(g)\right|: g(p)=0\right\}+\left|\phi_{n}(1)-1\right|,
\end{aligned}
$$

therefore by (7) and (11)

$$
\left\|\phi_{n}-\chi\right\| \rightarrow 0
$$

Hence we have for each sequence $\left\{\phi_{n}\right\}$ in $A^{\star}$ with $\left\|\check{\phi}_{n}\right\| \rightarrow 0$ and $\phi_{n} \rightarrow \chi$ weak* $(\chi \neq 0)$, that $\left\|\phi_{n}-\chi\right\| \rightarrow 0$, so by Theorem $3.1 A$ is AMNM.

\section{References}

1. S. A. Douglass, Introduction to mathematical analysis (Addison Wesley, 1996).

2. R. A. J. Howey, 'Approximately multiplicative maps between some Banach algebras', PhD Thesis, University of Newcastle upon Tyne, 2000.

3. K. Jarosz, Perturbations of Banach algebras, Lecture Notes in Mathematics 1120 (Springer, 1985).

4. B. E. Johnson, 'Approximately multiplicative functionals', J. London Math. Soc. (2) 34 (1986) 489-510.

5. E. J. McShane, 'Extension of range of functions', Bull. Amer. Math. Soc. 40 (1934) 837-842.

6. H. Whitney, 'Analytic extensions of differentiable functions defined in closed sets', Trans. Amer. Math. Soc. 36 (1934) 63-89.

7. H. Whitney, 'On the extension of differentiable functions', Trans. Amer. Math. Soc. 50 (1944) 76-81.

Richard Andrew Jonathon Howey

Department of Mathematics

University of Newcastle upon Tyne

Merz Court

Newcastle upon Tyne

NE1 7RU

Current address:

Department of Computer Science

University of Durham

Science Laboratories

South Road

Durham

DH1 3LE

r.a.j.howey@dur.ac.uk 